\title{
ÉPISTÉMOLOGIE, HISTOIRE ET HISTOIRE DES SCIENCES DANS LES ANNÉES 1930
}

\author{
1. L'ÉTRANGE THÉÂTRE \\ Enrico Castelli Gattinara
}

Résumé : Au début des années 1930, l'épistémologie, l'histoire et l'histoire des sciences ont eu une influence réciproque importante mais indirecte. Tant les philosophes que les historiens mettaient en jeu des concepts épistémologiques issus de la crise des sciences exactes et rejetaient l'empirisme et l'idéalisme au nom d'un rationalisme expérimental qui mettait en relation théorie et expérience. Il en résultait une conception dialectique de la science, essentiellement historique mais à propos de laquelle les historiens étaient encore timides du point de vue théorique. Désormais l'histoire était pourtant au cœur des réflexions philosophiques et épistémologiques.

MoTS-CLÉS : crise, épistémologie, expérience, histoire, raison, réalité, science, théorie.

ABSTRACT: During the 1930's epistemology, history and history of science reciprocally and importantly influenced each other, even if indirectly. Philosophers and historians discussed the epistemological concepts emerging from the crisis of the exact sciences. They rejected Empirism and Idealism for an " experimental Rationalism » that connected theory and experience. It all caused a dialectical conception of science, fundamentally historical, but for which historians were still reluctant from a theoretical point of view. History was nevertheless the centre of philosophical and epistemological reflexions.

KEYWORDS : crisis, epistemology, experience, history, reason, reality, science, theory.

Revue de synthèse : $4^{e}$ S. $\mathrm{n}^{\mathrm{0}} 1$, janv.-mars 1998, p. 9-36. 
ZuSAMmENFASSUNG: Um 1930 haben Epistemologie, Geschichte und Wissenschaftsgeschichte einen wichtigen, wenn auch nur indirekten Einfluß aufeinander ausgeübt. Sowohl Philosophen als auch Historiker haben über die epistemologischen Begriffe diskutiert, die aus der Krise der exakten Wissenschaften hervorgegangen waren. Sie haben Empirismus und Idealismus zugunsten eines experimentellen Rationalismus verworfen, bei dem Theorie und Erfahrung zusammengeführt wurden. Obwohl die dialektische Konzeption von Wissenschaft, die sich daraus ergab, in wesentlichen Punkten historisch war, waren die Historiker in der theoretischen Diskussion diesbezüglich eher zurückhaltend. Das änderte jedoch nichts daran, daß die Geschichte im Mittelpunkt der epistemologischen und philosophischen Überlegungen stand.

StICHWÖRTER : Krise, Epistemologie, Erfahrung, Geschichte, Vernunft, Wirklichkeit, Wissenschaft, Theorie.

Sommario: All'inizio degli anni 1930 l'epistemologia, la storia e la storia delle scienze hanno avuto un'importante, benché indiretta, influenza reciproca. Tanto $i$ filosofi che gli storici mettevano in gioco i concetti epistemologici emersi dalla crisi delle scienze esatte e respingevano l'empirismo e l'idealismo in nome di un razionalismo sperimentale che faceva interagire teoria ed esperienza. Ne risultava una concezione dialettica della scienza, fondamentalmente storica, nei confronti della quale tuttavia gli storici erano ancora timidi sul piano della teoria. Invece la storia era ormai al cuore delle riflessioni epistemologiche e filosofiche.

Parole ChIAvE : crisi, epistemologia, esperienza, storia, ragione, realtà, scienza, teoria.

Enrico Castelli Gattinara, né en 1959, directeur de la revue italienne Aperture. Punti di vista a tema, est maître de conférences associé à l'École des hautes études en sciences sociales, Paris. Spécialiste de la philosophie et de l'épistémologie françaises $\mathrm{du} \mathrm{xx}^{\mathrm{e}}$ siècle, il a publié Epistemologia e storia (Milan, Franco Angeli, 1996) et Les Inquiétudes de la raison (Paris, Vrin//EHESS, 1998).

Adresse : via A. Allegri da Correggio, 11, 00196 Rome, Italie.

Courrier électronique : aperture@iol.it <Enrico Castelli Gattinara> 
Durant les premières décennies du $\mathrm{xx}^{\mathrm{e}}$ siècle, plusieurs disciplines ont été bouleversées dans leurs fondements et dans leurs méthodes. Deux d'entre elles, l'épistémologie et l'histoire, ont eu aussi un rapport qui les a rendues dans quelques circonstances presque complémentaires, sous un certain point de vue. Dans l'état de crise où se trouvaient plusieurs formes du savoir, l'épistémologie d'une part et l'histoire de l'autre devaient établir des relations réciproques qui ne sont guère définissables de manière précise, mais qui ont permis des échanges à plusieurs niveaux.

Le rapport entre épistémologie et histoire date au moins du tournant du siècle. Or, bien qu'il ait changé au fil du temps, il n'a jamais réussi, curieusement, à être véritablement réciproque et direct. Pourtant, il ne s'agit pas ici de toute l'histoire de ce rapport (qui se prolonge d'ailleurs jusqu'à nos jours), mais seulement d'une petite partie de celle-ci, significative selon moi : celle des années 1930 ou, plus généralement, de l'entre-deux-guerres. Sans prétendre en donner une explication exhaustive, le présenter peut être un premier pas pour en tirer des éléments explicatifs.

Les philosophes des sciences, ceux qui s'intéressaient à l'épistémologie, étaient de plus en plus concernés par l'histoire, parce que celle-ci devenait essentielle pour une théorie de la connaissance soucieuse de prendre en charge l'évolution des sciences et des théories, évolution qui, à partir de la seconde moitié du $\mathrm{xIx}^{\mathrm{e}}$ siècle, devenait toujours plus rapide et triomphale. Grâce à la nécessité de revoir des concepts et des idées pour les rendre plus cohérents avec la nouvelle configuration des savoirs, ces philosophes ont en quelque sorte préparé le terrain sur lequel certains historiens mèneront par la suite leurs combats. Ainsi, Marc Bloch et Lucien Febvre le feront avec des « outils » et des « idées » issus de l'épistémologie et du débat philosophique de l'époque sur les conséquences des innovations théoriques et scientifiques qui bouleversaient la conception traditionnelle du savoir et les fondements de certaines disciplines importantes comme les mathématiques ou la physique. Cependant, ni les philosophes des sciences, si attentifs à l'histoire, ni les historiens, qui par la suite utilisèrent des concepts et des théories épistémologiques, ne s'inquiétaient de l'état du débat et des problèmes les plus actuels de la discipline qui leur était si utile.

Mais quelles théories épistémologiques les philosophes et les scientifiques posaient-ils au tournant de 1900 , et comment et pour quelle part ces théories se retrouvaient-elles dans les argumentations des directeurs des 
Annales? Dans les années 1930, en effet, les jeux étaient presque faits, du point de vue de l'épistémologie et de la crise des fondements théoriques des sciences, et les historiens des Annales, qui y étaient très sensibles, s'y référaient souvent dans leurs écrits. Tout semblait être remis en cause et l'indéterminisme en microphysique avait donné un coup presque définitif à la confiance que l'on avait dans les principes de la raison. Les philosophes et les savants cherchaient alors à tenir ensemble les débris des principes qui avaient semblé, jusqu'à la fin du siècle précédent, avoir régi la raison et la connaissance.

En témoigne un texte extraordinaire pour comprendre la stratégie de l'époque, où un philosophe et des scientifiques parmi les plus connus cherchaient à faire le point par rapport au grand bouleversement provoqué par les nouvelles théories : il s'agissait d'une série de conférences données en 1930 à l'École normale supérieure dans le but d'initier les jeunes aux problèmes les plus actuels des sciences et de la philosophie. Alexandre Koyré, qui en rendait compte en 1932, attirait cependant l'attention sur le rapport asymétrique qui persistait entre les philosophes et les scientifiques.

«On sait que les sciences [...] ont subi ces dernières années des transformations très profondes, fort importantes du point de vue philosophique, puisqu'entraînant [...] l'abandon des principes mêmes [...] du savoir scientifique, c'est-à-dire de la loi de causalité et de la détermination univoque des phénomènes de la nature'.»

Mais Koyré se lamentait en même temps du fait que les philosophes n'avaient pas été encore capables de fournir aux scientifiques les concepts nouveaux qu'ils leur demandaient et qui étaient requis par les nouvelles théories.

Or, ce reproche n'était pas tout à fait justifié, car certains philosophes et certains savants essayaient depuis le début du siècle - et même avant de suivre le pas de l'évolution des sciences, et quelques-uns, comme Ernst Mach, Henri Poincaré ou Pierre Duhem, avaient même commencé à préciser des conceptions et des principes nouveaux de la connaissance scientifique. L'épistémologie, qui naissait avec le siècle nouveau, constituait donc une sorte de réponse avant la lettre aux remarques de Koyré, bien que les philosophes rencontrassent effectivement beaucoup de difficultés à abandonner les vieux principes pour en trouver de nouveaux.

Quelles étaient donc les questions les plus importantes qui se posaient aux philosophes des sciences avant même la révolution einsteinienne? Les

1. Alexandre KoyrE, compte rendu, Revue philosophique, t. CXIV, 1932, p. 315 ; voir aussi, L'Orientation actuelle des sciences, Paris, Alcan, 1930. 
plus fondamentales — pour ne pas les retenir toutes - peuvent être rapportées à deux grands regroupements.

Le premier regroupement pourrait être classé sous la question du rapport entre nécessité et contingence (dont Émile Boutroux fut un des critiques les plus lucides). L'un des problèmes posés était la remise en cause, encore partielle au tournant du siècle, d'une des catégories essentielles de la raison humaine, la causalité. Avec celle-ci, un autre principe méthodologique fondamental était en question - depuis quelque temps déjà, à cause des problèmes mathématiques en jeu dans le calcul des probabilités —, à savoir celui de l'exactitude, si important pour l'identification des sciences dites « exactes », car l'exactitude probabiliste n'est certainement pas la même qu'une exactitude absolue. Et avec ce principe était suspecté aussi celui auquel, au début du siècle, presque personne ne songeait encore à toucher, mais qui était en réalité déjà affecté par une crise profonde, celui du déterminisme; pourtant, celui-ci allait être considéré encore longtemps comme le fondement inébranlable de tout procédé qui se prétendait scientifique, mais qui serait profondément secoué à partir de la seconde moitié des années 1920 par la conception probabiliste en microphysique ${ }^{2}$.

Le second regroupement serait placé sous le signe du rapport entre objet et sujet; mais les deux regroupements s'entrecroisent à plusieurs niveaux. Nous y rencontrons les efforts faits par la philosophie des sciences depuis la fin du XIX ${ }^{\mathrm{e}}$ siècle, en particulier par Mach, pour saisir la leçon kantienne contre l'empirisme positiviste; et c'est dans ce cadre que certains philosophes et scientifiques français ont commencé à développer une réflexion qui caractérisera longtemps l'épistémologie de langue française (du moins tout le long de la première partie du $\mathrm{xx}^{\mathrm{e}}$ siècle). Or, les problèmes impliqués dans la remise en cause du rapport de l'objet et du sujet sont très connus : il s'agit des rapports entre la connaissance et la réalité, la théorie et l'expérience, le fait et l'hypothèse. Le rôle que Poincaré a joué en ce domaine, par exemple, a été fondamental, et ses écrits d'avant la révolution einsteinienne témoignent du fait que ces problèmes étaient bien là avant les grands bouleversements apportés par les nouvelles théories physiques bien connus et auxquelles tous se rapportaient ${ }^{3}$.

Les épistémologies de Mach, de Poincaré ou de Duhem, malgré leurs profondes différences, partaient toutes de la remise en cause du fait brut et

2. Qu'il nous suffise de renvoyer au livre du mathématicien Émile Borel, Le Hasard, Paris, 1920, pour montrer que les problèmes du probabilisme affectaient déjà la conception traditionnelle du déterminisme, et que même ceux qui, comme Borel, restaient strictement déterministes, se voyaient obligés de poser la question et de défendre le déterminisme même avant la révolution de la mécanique quantique.

3. Le cas de Bachelard est emblématique à cet égard, car il fait commencer l'âge du « nouvel esprit scientifique » au moment de la parution du mémoire d'Einstein sur la relativité restreinte (1905), pas avant. Voir Gaston BaCHELARD, La Formation de l'esprit scientifique, Paris, Vrin, 1932, p. 7. 
de l'empirisme. Leur formule pourrait être résumée de la façon suivante : tout fait empirique, et notamment tout ensemble et toute série de faits empiriques n'existent qu'en fonction des hypothèses par lesquelles le scientifique les saisit. Toute saisie de faits, donc toute expérience réalisée à partir de certaines hypothèses, mène à une généralisation, et c'est seulement au niveau des généralisations qu'existent les sciences - car c'est seulement la généralisation qui permet la prévision, but de toute science pour Poincaré, et c'est par la prévision que l'on parvient à l'établissement des lois. Or, les généralisations se font par un langage purement conventionnel dont le critère est celui d'être le plus « commode » pour exprimer les lois qui y sont impliquées. Les faits empiriques en tant que tels et isolés entre eux n'intéressent personne : seule compte la relation, exprimée en langage conventionnel, que le scientifique trouve entre eux, la correction qu'il leur fait subir pour les accorder éventuellement aux hypothèses, etc.

Dans cette perspective, hypothèses et théorie sont toujours premières, et il est impossible d'expérimenter sans théorie préalable, sans idée préconçue; d'ailleurs, c'est toujours cette conviction que Poincaré explicite dans La Science et l'hypothèse en 1902. Le critère de l'objectivité scientifique était lui aussi profondément remis en cause, et on ne pouvait plus prétendre comparer naïvement les théories aux faits dits « extérieurs », ni croire que la vérité était cachée dans les objets et qu'il aurait fallu simplement la saisir en eux. La vérité et l'objectivité - deux concepts donc bien philosophiques que les scientifiques doivent toujours manier - devaient être conçues d'une façon différente, beaucoup plus dynamique que le critère traditionnel de la conformité ou de l'adéquation - cette question ne peut être développée ici car elle s'articule d'une manière très complexe et touche à la notion même de réalité ; sur ce sujet se creusait la différence par rapport à Duhem.

Une conception conventionnaliste et hypothétique de la connaissance et de l'expérience entraînait évidemment une conception non définitive des lois et des expériences elles-mêmes dans leur rapport à la réalité : rien n'empêche, disait Poincaré, de trouver d'autres conventions plus « commodes » pour rendre compte d'expériences nouvelles, par exemple celles de la relativité ou des géométries non euclidiennes, à propos desquelles pourtant il restait sceptique justement en ce qui concernait leur «commodité » ${ }^{4}$. Dès lors, les théories physiques étaient «considérées comme des libres créations de notre esprit, résultant d'une intuition presque poétique » - comme le disait Karl Popper — et l'expérience, loin

4. Henri Poincaré, La Science et l'hypothèse, $\mathrm{I}^{\mathrm{re}}$ éd. 1902, Paris, Flammarion, 1968, p. 76 : «Une géométrie ne peut pas être plus vraie qu'une autre; elle peut seulement être plus commode." 
d'être une accumulation mécanique d'observations, était essentiellement créative, car elle était le résultat d'interprétations libres, créatives et audacieuses $^{5}$.

Cela menait enfin à une remise en question de ce qu'on pouvait considérer comme « science » et de ce qui ne l'était pas, car les conséquences du conventionnalisme, par exemple, pouvaient conduire à un nominalisme : ainsi Édouard Le Roy, dans son extrémisme, rapportait tout à une sorte de liberté arbitraire du sujet. Poincaré se gardait bien d'une telle dérive, mais il savait que la conception de la science pouvait en être affectée ${ }^{6}$. Dans le cas du formalisme de Duhem, par exemple, la tendance réaliste que Henri Poincaré et Abel Rey cherchaient à garder dans la science était presque complètement abandonnée (d'où une polémique véhémente entre Duhem et Rey).

Toujours est-il que le rôle que l'on reconnaissait désormais aux hypothèses devenait décisif pour reformuler la théorie de la connaissance scientifique. Rey, qui pourtant se rangeait encore, dans une attitude polémique, du côté du positivisme, admettait dans ses écrits que l'objet consistait « en des relations posées par l'interprétation de l'expérience et par l'intelligence, par leur adaptation réciproque ${ }^{7} »$. L'objectivité de la physique était « relationnelle » tant pour Poincaré (« ce qui est objectif doit être commun à plusieurs esprits [...] : pas de discours, pas d'objectivité » et « la seule réalité objective, ce sont les rapports des choses ${ }^{8}$ ) que pour Rey. Il s'agissait en effet d'une objectivité discursive et conceptuelle, qui restait de toute façon strictement liée à l'expérience. Mais cette expérience n'avait plus rien d'élémentaire, parce qu'elle se faisait en fonction d'idées et d'hypothèses qui en dirigeaient le cours.

Henri Poincaré, Pierre Duhem, Édouard Le Roy, Abel Rey, Léon Brunschvicg, Émile Meyerson étaient tous d'accord sur ce point : les lois des sciences sont une représentation, une interprétation de l'expérience sur laquelle la réalité agit avec autant de force que la théorie qui l'engendre. D'où le fait que les lois changent, parce qu'elles ne peuvent être que des approximations d'une vérité qui nous échappera toujours; jamais elles ne

5. Karl Popper, Conjectures and refutations, Londres, Routledge and Kegan Paul, 1969, en part. chap. vill, « Le statut de la science et de la métaphysique », p. 330-331.

6. Voir H. PoINCARÉ, op. cit. supra n. 4, p. 24-25: "Quelques personnes ont été frappées de ce caractère de libre convention qu'on reconnaît dans certains principes fondamentaux des sciences. Elles ont voulu généraliser outre mesure et en même temps elles ont oublié que la liberté n'est pas l'arbitraire. Elles ont abouti ainsi à ce que l'on appelle le nominalisme et elles se sont demandé si le savant n'est pas dupe de ses définitions et si le monde qu'il croit découvrir n'est pas tout simplement créé par son caprice. Dans ces conditions, la science serait certaine, mais dépourvue de portée. »

7. Abel ReY, «La notion d'objet et l'évolution de la physique contemporaine ", Revue philosophique, vol. XCIV, 1922, p. 214.

8. H. Poincaré, La Valeur de la science, Paris, Flammarion, 1905, p. 262, 271. 
pourront en donner une détermination définitive et ultime ${ }^{9}$. Cela explique pourquoi la science évolue et pourquoi les théories se substituent les unes aux autres : l'histoire des sciences nous le montre aisément. Dans la perspective de cette épistémologie, l'histoire (l'histoire des sciences surtout) recouvrait un rôle de plus en plus essentiel pour tous ceux qui voulaient rendre compte de l'évolution des connaissances scientifiques. Qu'il s'agît de Duhem ou de Rey, de Brunschvicg ou de Meyerson, l'histoire donnait raison au mouvement dynamique de la connaissance impliqué par la remise en cause du rapport entre donnée et hypothèse.

La majorité des philosophes français des sciences restait convaincue de l'irréductibilité de l'expérience, mais d'une expérience qui a alors changé de statut, puisqu'elle construit l'objet à propos duquel elle se fait, et elle y parvient grâce à des hypothèses qui se structurent en théories; mais en retour, ces expériences, qui par leur aspect particulier et concret ont toujours quelque chose (des contingences) qui échappe à la théorie, peuvent de temps en temps obliger la théorie à modifier en partie les hypothèses initiales qui les avaient dirigées, ce qui à son tour va engendrer de nouvelles expériences, et ainsi de suite. Le caractère conventionnel et hypothétique des théories n'empêche pas l'existence d'une « réalité », mais cette réalité n'existe qu'en rapport avec la connaissance qui l'affecte. Et si, selon la leçon de Kant, nous ne pouvons connaître que des phénomènes, c'est parce que raison et réalité n'existent pas de façon indépendante mais sont fonction l'une de l'autre. La réalité, dont le statut est lui aussi relationnel - car elle n'est pas conçue comme une matérialité inerte ou existant pour soi indépendamment de tout -, n'est pas réduite à la raison qui la pense, comme dans le cas de l'idéalisme, parce qu'elle représente l'instance par laquelle la raison elle-même change et s'assouplit.

La conception dont nous parlons a été nommée le rationalisme expérimental, à la différence de l'idéalisme rationaliste ou du matérialisme empirique. Cette conception, qui se fondait sur un « rationalisme assoupli et fécond », selon une heureuse formule de Brunschvicg, se dérobait autant à la rigidité absolue et systématique de l'idéalisme, qu'à la naïveté objective du matérialisme : la connaissance était ce par quoi la raison et la réalité se rapportaient réciproquement. $\mathrm{Ni}$ l'une ni l'autre n'existaient plus comme instances autonomes, mais étaient les deux «pôles » du dynamisme de la connaissance elle-même. Pour Federigo Enriques,

9. Ibid., p. 1 : pour Poincaré la vérité « est un fantôme qui ne se montre à nous un instant que pour fuir sans cesse, qu'il faut le poursuivre plus loin et toujours plus loin, sans jamais pouvoir l'atteindre "; Federigo EnRIQuES, mathématicien et philosophe, répétait le même concept trente ans plus tard, in Signification de l'histoire de la pensée scientifique, Paris, Hermann, 1934, p. 7 : «La vérité n'est qu'un acheminement vers le vrai »; et toute la théorie de la connaissance approchée de Bachelard suivait le même sillage. 
«le processus constructif de la Science peut être comparé au mouvement d'une balançoire, où celui qui y est assis s'efforce de se pousser le plus en avant possible; à toute poussée en avant correspond une oscillation par laquelle le mouvement en arrière devient lui aussi plus prononcé, et cela rend la poussée toujours plus efficace. La Science, considérée dans son aspect génétique, ne monte pas seulement vers une objectivité toujours majeure, mais par contraste elle pousse à des sommets plus élevés la subjectivité des représentations, qui sont son mode de conquête ${ }^{10} »$.

La réalité a un sens grâce à la raison qui la « construit » chaque fois, mais la raison n'existe comme telle que par cette réalité inépuisable qui s'y oppose et qui l'oblige à rester toujours en mouvement. D'où, encore une fois, le caractère inéluctablement approché de notre connaissance scientifique : «l'ordre naturel de l'histoire », comme l'écrivait Brunschvicg, nous montre donc que la marque spécifique de notre intelligence consiste dans sa «capacité indéfinie de progrès [et dans son] inquiétude perpétuelle de vérification ».

C'était le mouvement dynamique et dialectique de la connaissance qui, de par ce fait même, était historique. Rey l'expliquait de la façon suivante :

« Il faut voir la pensée sous son aspect historique, et l'histoire sous un aspect dynamique [...] Connaître, c'est bien construire l'objet, mais c'est aussi, et en même temps, du même coup, apprendre comment l'objet est construit, car cette construction ne se fait ni par ni pour un esprit étranger qui cherche à s'y adapter du dehors ${ }^{11}$. ”

Et Brunschvicg, à propos de la vérité, le confirmait : «La vérité de la science n'implique plus alors la supposition d'une réalité transcendante : elle est liée aux procédés de vérification ${ }^{12}$.»

Il devenait dès lors légitime de se demander : qu'est-ce qu'une science? Et notamment : qu'est-ce qu'une connaissance scientifique, une fois qu'on est obligé de suivre les procédés de vérification et le jeu continu, sans cesse renouvelé, de la théorie et de l'expérience? La réalité que la science était censée expliquer et dévoiler se retirait dans un milieu flou et mouvant, instable, qui nous oblige à n'être jamais satisfaits de ce que nous croyons avoir atteint. Elle devenait ainsi problématique, et des philosophes comme Brunschvicg et Meyerson, ou même Bergson, se trouvaient dans la nécessité de repenser le réel.

10. F. Enriques, Problemi della scienza, Bologne, Zanichelli, 1906, p. 26.

11. A. ReY, Le Retour éternel et la philosophie de la physique, Paris, Flammarion, 1927, p. 288.

12. Léon Brunschvica, Les Étapes de la philosophie mathématique, Paris, Presses universitaires de France, 1912, p. 561. 
L'épistémologie bachelardienne qui se développait dans les années 1930 suivait le même ordre de problèmes, tout en en radicalisant certains aspects. L'énoncé de Gaston Bachelard de 1934, selon lequel «l'esprit aurait une structure variable, du fait que la connaissance a une histoire ", ne rompait donc pas avec la tradition épistémologique qui le précédait. De même, lorsque Bachelard affirmait qu'il n'y avait pas de donnée immédiate, parce que toute donnée était toujours un résultat, nous savons d'où il recevait ces idées. Nous ne nous étonnons pas non plus devant le constat que « l'expérience, dans la science contemporaine, n'est plus une constatation première », car nous savons que le statut du réel avait changé et que les sciences étaient des formations discursives dont le point de départ était toujours théorique. Cela ne veut naturellement pas dire que l'épistémologie bachelardienne était bel et bien une continuation de celle des philosophes de la génération précédente; mais il y avait une convergence problématique autour des questions que nous avons très synthétiquement rassemblées dans deux grands regroupements. Et le rôle toujours plus important que l'histoire allait avoir pour les épistémologues, notamment pour Bachelard qui en faisait même une théorie, était strictement lié à la remise en cause des concepts et des notions fondamentaux, y compris ceux de "science » et de « réalité » déjà évoqués.

La prise en charge de l'histoire de la part de l'épistémologie, provoquée par la crise où se trouvaient les diverses formes du savoir et de la raison, a donc été une nécessité théorique et pratique bien avant Bachelard. La science, renvoyant à son histoire, ne devait plus être statique et les critères de son identité restaient à jamais mouvants. Cela veut dire que dans les premières décennies $d u \mathrm{xx}^{\mathrm{e}}$ siècle entrait décidément et irréversiblement en crise l'identité de la science tout court (en langage poppérien, c'est le problème de la démarcation entre science et non-science) : les épistémologues et les philosophes étaient mobilisés soit pour formuler une nouvelle identité de la science capable d'intégrer l'histoire dans la formulation elle-même, soit pour assumer la « leçon " de l'histoire et fournir une solution qui faisait table rase de toute identité et de tout principe fondateur, soit encore pour s'engager dans une voie moyenne entre les deux. Pour tous, l'histoire était une réalité qu'aucune réflexion ne pouvait désormais ignorer, fût-ce pour en nier l'importance, comme dans le cas du néo-positivisme du Cercle de Vienne.

Or, si nous passons aux questions qui hantaient les historiens presque à la même époque, nous sommes frappés par un problème qui est strictement lié à ce que nous venons d'exposer. Il est en effet remarquable d'observer à quel point aussi bien Febvre que Bloch, dans le sillage de Henri Berr et de Paul Lacombe, revendiquaient pour l'histoire le statut de science. À plusieurs reprises, ils faisaient référence à la science comme méthode rigou- 
reuse et ils utilisaient le vocable « scientifique » pour indiquer ce qui caractérisait une recherche et un travail bien fait.

La «science " était évidemment le discours dominant, le discours par lequel on atteignait la vérité, le discours bien ordonné et notamment la manifestation du niveau le plus haut qu'avait atteint la culture humaine. Que l'histoire veuille être une science, cela n'avait rien de scandaleux en soi, à la condition de s'entendre sur le mot « science ». La question n'allait pas de soi, à l'époque (de même, d'ailleurs, qu'aujourd'hui), et il y eut un conflit au tournant de 1900 entre les tenants de l'histoire-science (Lacombe, Berr) accrochés aux modèles de la sociologie comme à ceux qu'ils tiraient d'autres sciences humaines et naturelles, les tenants de l'histoire "positive» et érudite, historisante (Gabriel Monod, Paul Harsin, Charles-Victor Langlois et Charles Seignobos), et les tenants d'une histoire non assimilable à aucune science (Charles Péguy, mais en partie aussi Alexandru Dimitri Xénopol). Ce conflit nous permet de comprendre deux choses : d'abord que les historiens n'étaient pas d'accord entre eux sur la méthode à suivre et concevaient leur discipline de façons très diverses (ce qui arrivait d'ailleurs aussi à d'autres sciences humaines, comme la psychologie ou la médecine); ensuite, que l'idée qu'ils se faisaient de la science n'était aucunement homogène et que les modèles auxquels ils se rapportaient n'étaient souvent pas comparables. Enfin, l'image de la science que les historiens avaient au début du siècle était tellement différenciée et en partie contradictoire que l'on ne doit pas s'étonner si, par la suite - jusque dans les années 1930 - cette image était restée non pas « vague », mais certainement ambiguë.

Il faut d'ailleurs signaler qu'en plein débat sur l'histoire-science, il y avait aussi ceux qui, comme Henri Pirenne, avaient gardé une attitude sceptique et ironique par rapport au problème. Dans un compte rendu de 1898, à propos de l'Introduction aux études historiques de Langlois et Seignobos, paru la même année, Pirenne ne se montrait absolument pas scandalisé du fait que l'histoire n'eut pas été une science. Il y déclarait ne pas comprendre le «ton de mépris avec lequel ils trait[ai]ent l'histoire » et pourquoi «ils lui en v[oulai]ent de n'être pas à la hauteur des vraies sciences, et d'en être réduit à se servir d'observations mal faites et incontrôlables »; mais il ne comprenait pas non plus la véhémence de tous ceux qui, à l'inverse des deux auteurs, s'acharnaient à soutenir que leur travail d'historiens était bien "de la science " ${ }^{13}$. Ce fut seulement par la suite que Pirenne prit en quelque sorte parti pour la science, tout en gardant son scepticisme ironique, et ce fut pour cela qu'en 1923 (dans sa conférence

13. Henri Pirenne, compte rendu de Charles-Victor Langlois, Charles Seignobos, Introduction aux études historiques (1897), dans Revue de l'instruction publique en Belgique, Bruxelles, H. Lemertin éd., 1898, t. XLI, p. 36-38. 
inaugurale au $\mathrm{V}^{\mathrm{e}}$ congrès international d'Histoire dont le titre était « La méthode comparative en histoire $»)$, il pouvait déclarer concevoir la méthode comparative comme la seule méthode capable de permettre à l'histoire de devenir une science, l'affranchissant des idoles du sentiment et lui permettant d'apprécier «à leur degré précis de vérité scientifique » les faits étudiés.

Or, nous avons vu que l'épistémologie était mobilisée depuis la fin du XIX ${ }^{\mathrm{e}}$ siècle pour réagir à ce qui s'était passé dans les sciences exactes, et qu'en France elle avait assoupli la conception positiviste et rationaliste du moment à propos de la connaissance scientifique. Certains historiens, et parmi eux bien sûr aussi les deux directeurs des Annales, en étaient parfaitement conscients, et nous allons voir comment; mais, fait à ne certainement pas négliger, ils « glissaient » pourtant souvent vers une « image » de la science encore très traditionnelle.

Le problème, car problème il y a à l'égard de la science — dans le sens du discours d'autolégitimation scientifique que les historiens faisaient pour leur propre discipline - concerne l'attitude que les historiens ont eue envers celle-ci : une attitude que l'on peut qualifier de «soumission » intellectuelle, comme s'il s'agissait d'une sorte de complexe d'infériorité à l'égard des sciences dures, exactes, qui leur semblaient non seulement très difficiles à comprendre, mais décidément plus propres à l'établissement objectif et méthodologiquement rigoureux des vérités qui les concernaient. Cette espèce de complexe a certainement interdit pour longtemps un échange productif et constructif avec cette forme particulière de l'histoire qui s'occupait spécialement des sciences (des sciences exactes, naturellement, vu que les sciences humaines venaient de s'établir comme telles); car, depuis le début du siècle, l'histoire des sciences restait décidément séparée de toutes les autres formes de l'histoire. Ce n'est donc pas un hasard si, du point de vue institutionnel, elle était enseignée seulement dans les facultés de philosophie: les philosophes voulaient dominer et même $s$ 'approprier cette branche de l'histoire, alors que les historiens généralistes, peu intéressés, ne s'interrogeaient pas trop à son sujet.

Cependant, dans la conjoncture intellectuelle des années 1930, l'histoire des sciences aurait pu donner aux historiens une image nouvelle et plus problématique des sciences. Elle leur aurait ainsi permis de franchir sans hésiter la ligne de l'épistémologie, c'est-à-dire d'en faire eux-mêmes, d'en traiter sans complexe et surtout sans craindre de retomber par là dans un nouveau dogmatisme. Ce dernier problème était, en effet, l'un des plus graves pour les historiens des Annales, et il influençait leur attitude à l'égard de la philosophie; car c'était de cela qu'ils avaient surtout peur : ils ne voulaient plus risquer d'être dominés par n'importe quel dogmatisme philosophique - ce qui pourrait contribuer à expliquer aussi leur méfiance 
à l'égard du marxisme -, d'où la formule sans appel de Febvre : "Philosopher - ce qui, dans une bouche d'historien signifie [...] le crime capital $^{14}$.»

L'histoire des sciences, en effet (mais il faudrait peut-être dire « une certaine histoire des sciences", celle de Hélène Metzger et d'Alexandre Koyré par exemple), avait saisi le bouleversement philosophique et épistémologique de l'époque et avait pratiqué la discipline en conséquence, renforçant ainsi le rôle qu'elle avait joué pour les philosophes sans pour autant se soumettre à leur hégémonie. Le cas de Metzger me semble exemplaire à cet égard, car il montre comment une historienne, en tant qu'historienne, a pu introduire les innovations épistémologiques et théoriques dans la pratique même de ses travaux, sans que ceux-ci perdent en « rigueur historique ou documentaire ». Or, ni Bloch ni surtout Febvre ne semblaient s'en apercevoir, car ils gardaient une attitude méfiante à l'égard de l'histoire des sciences, encore trop érudite ou trop philosophique pour eux. De toute façon, elle ne leur semblait absolument pas «sociale », puisqu'elle n'était pas ancrée dans l'humain vivant et collectif ${ }^{15}$.

J'ai dit « Febvre surtout », parce qu'il fréquentait bien plus assidûment que Bloch le Centre international de synthèse de Berr - participant même à sa direction - dans lequel travaillaient et discutaient les historiens des sciences de la section d'Histoire des sciences que Berr avait voulu leur consacrer spécialement. Il aurait donc pu être au courant de ce que ces historiens étaient en train de tenter à côté de lui : les interventions les plus intéressantes de Metzger, secrétaire de la Section, avaient en effet lieu dans les réunions périodiques de la Section tenues au Centre et étaient ensuite publiées par la revue Archeion, qui en était l'organe. Mais il n'en fut pas ainsi. Il aurait pu, dans le cas contraire, se rendre compte que certains historiens des sciences étaient en train de se charger du même effort, qu'ils combattaient les mêmes ennemis, ou presque, et notamment qu'ils auraient pu s'entraider et exercer une influence bénéfique mutuelle, ainsi qu'ils le réclamaient ouvertement mais en vain.

Un phénomène bien étrange, sinon paradoxal, se produisit : il y avait, d'un côté, des textes de Febvre qui souhaitait plus de collaboration entre historiens et historiens des sciences et qui invitait même à constituer une véritable histoire des sciences, comme si elle n'existait pas encore; mais il existait, d'un autre côté, des textes où des historiens des sciences (Rey et

14. Lucien Febvre, Combats pour l'histoire, Paris, Armand Colin, 1953, p. 433.

15. Telle est la situation dans les années 1930, mais par la suite l'attitude des historiens par rapport aux historiens des sciences changera partiellement, bien qu'ils gardent toujours une grande méfiance envers la philosophie et les grandes abstractions, ainsi que le montre la polémique entre Febvre et Koyré en 1950, sur laquelle Pietro REDONDI a attiré l'attention dans son article "Science moderne et histoire des mentalités. La rencontre de Lucien Febvre, Robert Lenoble et Alexandre Koyré », Revue de synthèse, t. CIV, 111-112, 1983, p. 324-330. 
Metzger, par exemple) souhaitaient plus de collaboration avec les historiens généralistes pour donner plus d'élan et de force à leur propre discipline. L'exigence était donc la même et aurait pu engendrer un effort commun qui pût donner à l'histoire des sciences toute l'importance dont elle avait encore besoin pour ne plus rester sous la domination de la philosophie. Or, les historiens d'un côté et les historiens des sciences de l'autre ne semblèrent pas s'apercevoir de ce terrain commun et restèrent sourds aux interpellations réciproques.

Cela est d'autant plus remarquable de la part de Febvre qu'il travaillait à plusieurs projets (institutionnels ou personnels, tels par exemple l'Encyclopédie française ou son livre sur Rabelais) qui touchaient d'une façon ou d'une autre l'histoire des sciences.

Mais les rapports avec ceux qui s'occupaient de cette forme de l'histoire n'étaient pas faciles, notamment au sein du Centre international de synthèse, pourtant créé pour faciliter la collaboration interdisciplinaire, comme Berr le répétait presque à chaque séance et à toute assemblée générale. La section d'Histoire des sciences jouissait d'une certaine indépendance par rapport aux autres sections du Centre, et ses séances étaient fréquentées presque exclusivement par des historiens des sciences. Cette Section avait d'ailleurs son propre organe, Archeion, et tous deux étaient dirigés par Aldo Mieli, qui les considérait comme ses créatures et sa propriété. Dans la revue, on rapportait fidèlement tout le travail de cette section, alors que les activités des autres sections étaient relatées dans l'organe du Centre, la Revue de synthèse. Ceux qui s'intéressaient à l'histoire des sciences ne devaient donc consulter qu'Archeion. Or, Febvre ne s'y intéressait apparemment pas beaucoup et il n'avait certainement été séduit ni par la revue ni par son directeur : c'est du moins ce qui saute aux yeux si l'on compare l'attitude de Febvre à l'égard d'Archeion, dont il n'est jamais question dans les Annales, et celle qu'il eut envers une autre revue d'histoire des sciences fondée en 1934 et dirigée par Rey, Thalès, qui allait bénéficier de trois comptes rendus dans les Annales entre 1934 et 1939.

Certes, on pourrait rétorquer que l'attitude d'Archeion et de Mieli à l'égard de l'histoire générale, et en particulier à l'égard des efforts d'innovation pratique et méthodologique que Febvre et Bloch étaient en train de réaliser par leur revue, était elle aussi d'indifférence complète. D'ailleurs, Febvre fit également ce reproche à Thalès, qu'il jugeait trop concentrée sur sa spécificité disciplinaire et sur les questions philosophiques impliquées. Et même si cela n'explique ni ne justifie encore rien, il est important de le constater pour comprendre ce qui était en train de se passer à l'époque.

À part Thalès, les Annales ignoraient d'ailleurs aussi une autre revue très importante d'histoire des sciences, Isis, née en 1913 et dirigée par George 
Sarton. Bien qu'en langue anglaise (mais publiée en Belgique), cette revue fut bien accueillie en France dès ses débuts. Elle fit souvent paraître plusieurs articles d'historiens et de philosophes des sciences français, notamment de ceux qui s'intéressaient au lien entre l'histoire des sciences et l'histoire des idées.

Au milieu des années 1930, par conséquent, alors que Febvre rappelait que, dans les Annales, il avait « souvent déploré l'état d'abandon dans lequel végétaient chez nous les études d'histoire des sciences », il y avait au moins trois revues qui opéraient dans le domaine et qui s'efforçaient de rassembler les travaux des chercheurs les plus érudits et les plus novateurs.

En vingt ans, de 1929 à 1948, les Annales consacrent seulement 15 textes à l'histoire des sciences et aux questions de « science», dont 14 sont des notes critiques ou des comptes rendus (le seul article de fond qui y est en partie consacré porte un titre déjà en soi significatif : «Arts et sciences, témoins de l'histoire sociale », de Pierre Abraham, en 1931). Des 14 notes ou comptes rendus cités, 5 ont paru entre 1929 et 1936, 7 entre 1937 et 1939, 2 entre 1940 et 1948 . Or, la plupart de ces écrits portaient sur des questions d'histoire des techniques, et il n'y en avait aucun sur l'histoire des sciences proprement dite.

Ce qui intéressait les deux directeurs, c'étaient en effet les techniques matérielles, agraires, commerciales, financières, industrielles, économiques en général, etc.; ce n'étaient pas les techniques abstraites ou concrètes utilisées par les différentes sciences, qui n'avaient apparemment aucun rapport au « social », si ce n'est, à la rigueur, en deuxième instance. Ainsi, parmi les quelque 250 articles, notes et comptes rendus consacrés en vingt ans à l'industrie, 24 seulement étaient consacrés aux techniques (techniques productives, histoire des techniques, etc.) ${ }^{16}$.

Febvre et Bloch n'étaient donc pas intéressés par l'histoire des sciences que l'on faisait autour d'eux : elle était trop éloignée de leurs intérêts

16. Bien qu'il ait commencé à publier ses travaux d'histoire des sciences vers la fin des années 1930, aucun livre de Koyré n'est jamais présenté dans les Annales. Il en va de même avec Bachelard, qui n'eut droit à un compte rendu signé par Febvre qu'en 1939, à propos d'un livre, La Psychanalyse du feu, qui avait pourtant moins d'importance que les ouvres épistémologiques qu'il avait publiées auparavant. Aucune référence n'est d'ailleurs faite ni à L. Brunschvicg, ni à É. Meyerson, ni à H. Metzger. Même A. Rey n'a droit à être commenté qu'en 1946, lors de la parution de ses travaux posthumes sur la science grecque. Il est étonnant aussi de noter que, alors que les Annales restent presque totalement imperméables aux questions et aux ouvrages philosophiques durant les années 1930 (2 écrits sur le sujet), dans les années 40 , une vingtaine de notes ou de comptes rendus y sont consacrés (mais aucun ne traite de philosophie des sciences ou d'épistémologie). 
immédiats et pratiques, elle était trop imprégnée de philosophie ${ }^{17}$, trop spécialiste et intellectuelle, et en fin de comptes trop abstraite. L'histoire des sciences traitait des sciences exactes - argument fort difficile - sans impliquer les hommes, les pratiques humaines, les laboratoires humains, enfin la vie humaine qui les faisait vivre. À l'époque, on faisait souvent l'histoire des sciences d'une façon dite « internaliste » (ce qui, en paraphrasant Febvre, dans la bouche d'un historien des Annales aurait été le crime capital) : on ne s'intéressait que très peu à la psychologie du savant, aux contraintes sociales, aux moyens techniques de production, aux intérêts idéologiques, etc. Cette attitude allait d'ailleurs soulever une querelle formidable dans les années de l'après-guerre entre internalistes et externalistes - comme Robert K. Merton et Michael Polanyi entre autres —, querelle qui ne s'est pas encore apaisée aujourd'hui, malgré les efforts de la sociologie des sciences.

Or, il est évident que cette façon internaliste de faire l'histoire ne pouvait pas satisfaire nos historiens ${ }^{18}$. Mais ils auraient pu s'intéresser du moins à ceux qui, épistémologues ou non, essayaient d'interpréter l'histoire des sciences dans le cadre d'une histoire plus générale des idées, tels Metzger, Koyré et en partie Bachelard, vu que l'histoire des idées était l'objet d'une très grande attention tant de la part de Bloch que de la part de Febvre.

Dans une lettre que Febvre écrivait à Berr, le 2 octobre 1928 (les travaux de Bachelard, Metzger et Koyré n'étant pas encore parus), à une date donc où le projet des Annales auquel il pensait avec Bloch depuis six ou sept ans était enfin mûr, la grande disponibilité de Febvre envers l'histoire des idées est évidente et confirme ainsi la stratégie de l'historien qui venait de publier, la même année, son livre sur Luther où l'histoire des idées tenait justement une place importante. Ce genre d'histoire n'était donc pas étranger aux directeurs de la revue dont le premier numéro allait paraître en janvier 1929. Mais dans cette lettre à Berr, Febvre écrit aussi autre chose, qui nous intéresse directement ici :

«Si je puis mettre sur pied cette Revue d'histoire économique convenablement [...] j'aurai, je crois, accompli une bonne cuvre. - Il y en a une autre qui

17. En effet, même Metzger s'adresse plutôt aux philosophes qu'aux historiens, et la façon dont elle présente les problèmes d'histoire de la chimie, ou d'histoire des sciences en général, tient surtout compte des questions philosophiques qu'elles auraient pu soulever.

18. Rappelons que l'histoire « internaliste » des sciences n'était pas le seul genre d'histoire que l'on faisait dans les années 1930 : dans le célèbre II $^{e}$ congrès international d'Histoire des sciences (Londres, 1931), des historiens soviétiques avaient scandalisé et séduit l'auditoire en proposant une interprétation marxiste de l'histoire des sciences, ce qui permettait de considérer les Principia de Newton du point de vue du matérialisme historique (Boris Hessen). Le débat suscité par les interventions des soviétiques avait été reporté dans Archeion, 14, 1932. 
m'apparaît singulièrement désirable, elle aussi. C'est la constitution d'une Revue d'histoire des idées. La matière et l'esprit. Vous savez combien je souhaiterais que la Revue de synthèse, je ne dis pas se transforme, car depuis longtemps elle est orientée dans ce sens, mais prenne enfin cette place qui est à prendre, en France et à l'étranger ${ }^{19}$, "

Prenons garde aux métaphores employées, car elles révèlent ce que j'ai affirmé plus haut à propos de l'image de la science à laquelle les historiens restaient liés : «La matière et l'esprit. » La matière, c'étaient les Annales, l'histoire militante pure et dure, celle qu'on faisait avec les mains sales de la poussière des archives et avec l'œil vigilant aux traces non écrites de la culture matérielle. C'était celle des enquêtes, des longues analyses, des fouilles, des problèmes bien délimités, des pratiques. L'esprit, c'étaient les idées, les théories, les grandes synthèses, les mentalités. C'était ce qui venait avant le travail concret de l'historien, et c'était surtout aussi ce qui suivait l'effort d'analyse.

La matière et l'esprit : ce partage très classique impliquait une division du travail que la revue de Berr et les Annales ne pouvaient pas, en principe, accepter! Mais la conception de l'esprit, comme synthèse intellectuelle qui suit le travail matériel de l'analyse, long et fatiguant, se retrouve également chez Bloch (qui gardait, lui aussi, une conception en partie assez traditionnelle du travail scientifique). "Pratiquement, comment travailler? ", se demandait-il dans son magnifique essai sur l'histoire comparée de 1928.

«Il va de soi que la comparaison n'aura de valeur que si elle s'appuie sur des études de faits, détaillées, critiques et solidement documentées. Il n'est pas moins évident que l'infirmité des forces humaines interdit de rêver, pour les recherches de première main, des cadres géographiques ou chronologiques trop vastes. Fatalement le travail comparatif proprement dit sera toujours réservé à une petite partie des historiens [...] Ne nous le dissimulons pas néanmoins: comme les études particulières sont encore, dans beaucoup de domaines, très peu avancées, il ne pourra lui-même avancer que très lentement. C'est toujours le vieux mot : des années d'analyse pour une journée de synthèse [selon la formule de Fustel de Coulanges]. »

La récolte des faits, le long travail matériel pour repérer les documents, c'est la matière, l'expérience empirique. Bloch s'en rendait bien compte, et

19. Lettre de L. Febvre à H. Berr, 2 oct. 1928, partiellement citée par Bertrand MülLER dans son introduction à Marc Bloch, Lucien Febvre, Correspondance, Paris, Fayard, 1994, p. XLAı (c'est moi qui souligne), repr. in L. Febvre, Lettres à Henri Berr, prés. et annot. par Jacqueline Pluet et Gilles Candar, Paris, Fayard, 1997, p. 337. Dans cette lettre, L. Febvre explicite l'enjeu stratégique qu'il envisage par la complémentarité entre les Annales et la Revue de synthèse historique, ce qui le pousse à maintenir un rôle très important dans la direction de la revue de Berr. 
savait que s'il s'en tenait là, en 1928, son discours risquait de tomber dans le cadre d'un mode vieilli et périmé de faire «science ", inductif et empiriste. C'est pourquoi il y ajoutait tout de suite après l'esprit : " Mais on cite trop souvent cette maxime sans y ajouter le correctif nécessaire : l' " analyse " ne sera utilisable pour la "synthèse " que si, dès le principe, elle l'a en vue et se préoccupe de la servir ${ }^{20}$. » Parce qu'il faut bien des hypothèses pour guider la recherche (comme Bloch l'écrit dans une page suivante). Là aussi persiste une image traditionnelle de la science, car dans les mots de Bloch nous assistons à ce qui caractérise assez souvent l'attitude des historiens à l'égard de la révolution épistémologique, attitude ambiguë, où l'on s'efforce de " corriger ", d'ajouter des « correctifs » aux énoncés traditionnels, sans jamais les abandonner définitivement. Ce comportement ne se limite pas évidemment aux historiens, et il est même plus présent chez les sociologues ou les spécialistes d'autres sciences humaines de l'époque, pour ne pas parler de la majorité des savants qui opéraient dans les sciences naturelles et des philosophes eux-mêmes : pourtant, il me semble intéressant de constater la rencontre entre l'effort d'innovation épistémologique et la persistance des vieilles conceptions de la science; cette situation manifeste le trouble, la «crise » et l'état mouvant dans lequel se trouvaient les idées dans l'entre-deux-guerres. En ce qui concerne l'histoire «nouvelle manière », il est encore plus extraordinaire de voir que Bloch était parfaitement conscient de la difficulté d'abandonner les procédés et les cadres traditionnels de la discipline, et de suivre la « révolution » pratique et méthodologique proposée par les Annales : «Dans ce que nous avons entrepris, il y a au fond, une espèce de petite révolution intellectuelle : il est difficile d'y plier les autres, et de nous y plier nous-mêmes ${ }^{21}$. »

Peut-être, si l'attention des historiens s'était aussi portée sur les questions épistémologiques en cours d'élaboration à l'époque, cette difficulté aurait-elle pu être moins importante et les Annales auraient-elles pu abandonner en toute tranquillité leur strict refus de tout propos théorique, méthodologique ou philosophique. Or, en dépit de la grande influence exercée par les nouvelles idées épistémologiques sur Febvre et sur Bloch - fait bien connu et rappelé dans les pages qui suivent - , ni l'un ni l'autre n'y avaient jamais prêté directement la moindre attention. Ceci est, en effet, une évidence à la lecture même de leurs textes car il n'y a pas trace des œuvres épistémologiques de Bachelard, de Metzger ou de Koyré dans les notes de lecture de Bloch, par exemple, ou dans les comptes ren-

20. M. BLoch, "Pour une histoire comparée des sociétés européennes », Revue de synthèse historique, t. XLVI, 136-138, déc. 1928, p. 15-50, repr. in ID., Mélanges historiques, 1963, Paris, Fleury/Éd. de l'École des hautes études en sciences sociales, 1983, p. $37-38$ (c'est moi qui souligne).

21. Lettre de M. Bloch à L. Febvre, 20 sept. 1929, in M. Bloch, L. Febvre, Correspondance, op. cit. supra n. 19, p. 205. 
dus publiés par Febvre dans les Annales et la Revue de synthèse durant les années 1930. Il en allait de même des réunions du samedi de Strasbourg, consacrées à la présentation critique des nouvelles parutions de librairie : plusieurs textes philosophiques y furent commentés, mais jamais des livres de philosophie ou d'histoire des sciences.

Recusant toute philosophie, nos historiens étaient alors obligés d'insister longuement sur leur propre révolution, ce qui n'aurait pas été un mal si leur conception critique et nouvelle ne s'était prêtée de temps à autre à faire réapparaître des vues traditionnelles comme, par exemple, cette fâcheuse division entre matière et esprit évoquée plus haut, ou encore le partage net et sans appel entre l'histoire économique et sociale et l'histoire des idées.

Ce partage me semble problématique et en fin de compte injustifié, car s'il est vrai que les Annales devaient garder leur spécificité disciplinaire et ne voulaient pas être une revue de méthode (tâche dévolue à la revue de Berr), il est tout aussi vrai qu'elles se proposaient depuis le début d'éliminer tout cloisonnement disciplinaire. Or, si tel était l'enjeu (même pour Bloch qui, par rapport à Febvre, était plus « historien d'atelier », au point que Febvre avait écrit de lui en $1929^{22}$ : « Mon codirecteur est très historien, et très érudit. Je l'ai laissé aller, un peu trop peut-être. »), pourquoi l'ouverture interdisciplinaire concernait-elle seulement les sciences humaines, à l'exclusion de la philosophie, de l'épistémologie, de l'histoire des sciences et même des sciences exactes?

Malgré les critiques que l'on pouvait légitimement faire à la philosophie des sciences du point de vue de l'histoire sociale, ces réserves à l'égard de l'épistémologie et des sciences exactes étaient dues en réalité à une conception encore très « disciplinaire » et conservatrice des domaines du savoir. Certes, l'air de supériorité et surtout la prétention d'hégémonie intellectuelle des épistémologues et des philosophes étaient insupportables (même Bachelard n'y échappait pas); mais des idées, des problèmes et des remises en question auraient dû beaucoup intéresser les historiens. Il y avait des buts en commun, il y avait un effort commun et même un esprit critique commun : enfin, d'une façon qui n'a jamais été manifeste et reconnue, une influence certaine s'exerçait des uns sur les autres (dans le cas de Febvre, par exemple, cette influence passait par Rey, avec lequel il était continûment en rapport depuis le début du centre de Berr, puis rue du Four ${ }^{23}$ ). Cependant, on peut se demander si cette influence a été comme refoulée et cachée pour éviter le risque de la domination de la philosophie sur les sciences, par rapport auxquelles l'histoire, selon une expression de Febvre,

22. Lettre de L. Febvre à Albert Thomas, 8 oct. 1929, citée in M. Bloch, L. Febvre, Correspondance, op. cit. supra $\mathrm{n} .19$, p. XLIII.

23. Siège de l'Encyclopédie française et de l'Institut d'histoire et de philosophie des sciences dirigé par Rey. 
se sentait encore comme une Cendrillon assise dans un petit coin, démunie de moyens pour affirmer son indépendance.

Ce véritable «complexe » à l'égard des sciences exactes et des autres sciences humaines, cette peur de tomber dans les méfaits que la philosophie (de l'histoire) causait par exemple en Allemagne, cette méfiance des idées abstraites (qui provoquera dans les années 1950 la polémique entre Febvre et Koyré) n'étaient pas justifiés de la part de nos historiens. Ils suivaient avec attention le débat philosophique qui leur était contemporain, ils en apprenaient même beaucoup, mais ils gardaient une distance infranchissable : en cela Febvre semble avoir eu bien plus de responsabilité que Bloch, qui s'en inquiétait moins. En revanche, les philosophes ignoraient totalement ce que faisaient les historiens, et même ceux qui avaient compris le rôle de l'histoire dans l'innovation et la dynamisation de la théorie de la connaissance ne s'interrogeaient pas sur les modifications que la discipline avait commencé à engendrer : ils se référaient ainsi souvent à des historiens et à des exemples qui appartenaient encore à une conception de l'histoire très traditionnelle, ce qui les empêchait de donner plus d'élan à leurs théories, comme ce fut le cas pour Meyerson ${ }^{24}$. Dans cet étrange rapport/non-rapport, les philosophes des sciences et les historiens avaient besoin les uns des autres, car ils saisissaient réciproquement les problèmes et les idées des autres, mais sans confrontation directe.

Une question se pose toutefois avant toute autre : les historiens avaientils le droit d'éviter tout problème épistémologique posé comme tel? Avaient-ils le droit de percevoir leur discipline comme une Cendrillon, comme une science prématurée, instable, incertaine de ses propres méthodes et de ses propres fondements? La réponse est non, de toute évidence, car non seulement toute science construit d'elle-même sa propre épistémologie, mais l'histoire des Annales se posait depuis le début, qu'elle le sût ou non, comme une révolution épistémologique des méthodes et des pratiques acquises.

Or, la réponse des historiens a pourtant été positive, celle de Febvre surtout, car ils avaient gardé une conception assez conservatrice du caractère scientifique, et du statut même de «science », que devait avoir une discipline, ce qui déclenchait leur complexe d'infériorité (le terme même d'infériorité, nous allons le voir, sera utilisé par Febvre en ce sens).

Le cas de Bloch a été en partie différent. Bien qu'il partageait les mêmes idées que Febvre, il était moins inquiet que son collègue aîné et il

24. Pour un approfondissement critique de la considération dans laquelle les philosophes tenaient l'histoire, tout en ignorant les efforts innovateurs internes à la discipline, voir E. CAStelli Gattinara, «Crise de la raison et pensée de l'ouverture », thèse de doctorat, dactyl., Paris, École des hautes études en sciences sociales, 1992, et ID., Epistemologia e storia, Milan, Franco Angeli, 1996, chap. II. 
reconnaissait la nécessité de prendre position d'un point de vue théorique et méthodologique. Il était en quelque sorte plus «disponible » envers les questions épistémologiques, et c'est en ce sens que l'on pourrait juger les efforts qu'il a faits pour écrire l'Apologie pour l'histoire: montrer par une œuvre «théorique » que la nouvelle façon de concevoir et de faire l'histoire était sur le même plan " scientifique » que les autres sciences, quelles qu'elles fussent, et cela même grâce à ce qui rendait l'histoire bien plus souple, problématique et pluraliste que les autres sciences.

Cette détermination méthodologique et stratégique de Bloch par rapport à Febvre est confirmée par son projet de publier en 1932 un livre qui devait rassembler ses articles de méthode, Historiens à l'atelier, dont le but était explicitement celui d'être une alternative aux traditionnels traités de méthode historique, aux manuels, etc. Ainsi qu'il l'expliquait à son éditeur, Bloch voulait faire comprendre aux lecteurs qu'il ne fallait plus se contenter de l'histoire belle et faite; au contraire, l'histoire, étant science du mouvement, toujours en devenir, ne pouvait plus en tant que telle prétendre à une codification fixée une fois pour toutes. Elle était une science « vivante ». D'autant plus, ajoutait Bloch, que montrer les différents points de vue qui se formaient successivement dans l'atelier d'un historien donnait mieux l'idée de la réalité problématique de la méthode historique. Offrant de l'histoire une vue plus dynamique et plus proche de sa pratique réelle, ce «traité de la méthode » aurait fourni à un large public une vision plus directe du travail des historiens (Febvre allait poursuivre la même stratégie avec ses Combats pour l'histoire, mais seulement vingt ans après).

Comment l'absence de confrontation critique et explicite, sans complexes, avec l'épistémologie (et l'histoire des sciences) a-t-elle rendu plus difficile la stratégie de la «nouvelle histoire», et l'a-t-elle surtout empêchée de soutenir la tête haute une confrontation avec les philosophes?

En 1922, à une date donc où il n'était plus un jeune historien, Febvre, discutant le caractère scientifique de la géographie physique et de la géographie humaine, constatait ceci : «L'histoire n'en est pas là. Elle ne croit même pas encore, tout entière, à sa qualité de science. Pour reprendre la même formule, elle ne fait pas figure de science à ses propres yeux », et il renvoyait en note au livre de Berr, La Synthèse en histoire, pour la discussion du problème ${ }^{25}$. Mais la conception que Berr avait de la science était véritablement très traditionnelle : il vaudrait la peine de s'y attarder,

25. L. Febvre, La Terre et l'évolution humaine, Paris, La Renaissance du livre, 1922, p. 38 (c'est moi qui souligne). Febvre considérait ce livre de Berr (Paris, Félix Alcan, 1911) comme "le seul livre de méthode » digne d'être lu par ses étudiants, voir L. FEBvre, « Problèmes de méthode : comment on écrit l'histoire ", Revue de synthèse, t. LIII, déc. 1933, p. 270. 
car cela pourrait aider à justifier les distances que prenait Febvre à partir de la fin des années 1930. Ce qui était en jeu, ici, c'était une image de la science à laquelle toute discipline semblait devoir s'adapter, si elle voulait prétendre à l'appellation de «science». Faire «figure de science à ses propres yeux », croire «à sa qualité de science »..., ce discours Febvre le prenait au sérieux, et il le maintint encore longtemps, car nous retrouvons l'image berrienne de la science encore très présente dans l'article « History ", qu'ils écrivent ensemble pour l'Encyclopaedia of the social sciences en 1931. Cependant, sauf quelques paragraphes et quelques phrases distribués ici ou là, Febvre n'expliquait nulle part ce qu'il entendait par « science » (et nous sommes à une période où, souvenons-nous en, l'épistémologie en faisait le sujet de ses réflexions).

C'est qu'il n'avait pas encore le « courage intellectuel » de proposer en tant qu'historien une nouvelle vue sur ce qu'était une science, bien qu'il en eût tous les outils (vocable qui lui était particulièrement cher). En effet, bien avant d'en avoir les outils matériels que lui donneront les Annales, il disposait déjà des outils intellectuels qu'il employait dans ses œuvres. L'histoire ne fait pas figure de science, avait-il écrit, et il ajoutait : " [...] de quoi sans doute elle n'a pas lieu de se féliciter naïvement, au contraire. Mais cette infériorité n'est pas sans quelque avantage ${ }^{26}$.»

L'avantage, aux yeux de Febvre, consistait dans le fait que l'histoire n'était liée à aucune doctrine (il pensait évidemment au déterminisme), à aucun corps de théories déjà établies «qui restreindrait sa liberté de jugement individuelle», et que donc elle pouvait étudier d'une façon « librement critique », « sans aucune rigidité dogmatique » des sujets que la géographie était par exemple obligée d'exposer selon une conception beaucoup plus rigide (le tableau). Encore une fois, les mots et les métaphores sont éclairants : nous avons en effet l'indication de l'infériorité de l'histoire (ce que j'ai appelé le « complexe »), ce dont elle n'avait pas « sans doute » le droit de se féliciter. Par contre, elle avait des « avantages». Febvre nous montre par là qu'il pouvait y avoir une alternative épistémologique dans la conception scientifique de l'histoire (liberté critique, souplesse, nondogmatisme, etc.), mais il ne la posait pas au même niveau scientifique. Fallait-il se réjouir de ces avantages de l'histoire ou non? Était-ce une infériorité ou pas? Febvre reste imprécis et ambigu à ce propos. Mais, avec Berr et les philosophes des sciences qu'il avait connus grâce à lui, il savait que le déterminisme était désormais insoutenable, que les explications mécanistes n'étaient plus satisfaisantes et que l'esprit critique devenait de plus en plus important d'un point de vue épistémologique (l'influence de Vidal de La Blache à ce sujet avait été elle aussi déterminante).

26. L. FebvRe, La Terre et l'évolution humaine, op. cit. supra n. 25, p. 38. 
On pourrait donner maints exemples du même genre, puisés dans les écrits de Febvre et de Bloch d'avant les années 1930. Qu'il suffise d'en citer un seul, de Febvre toujours, pourtant écrit en 1936, à une époque où les nouvelles implications épistémologiques de l'histoire lui étaient bien claires et l'empêchaient de tomber normalement dans les ambiguïtés qu'on vient de signaler; mais les vieilles images sont longues à mourir. Il s'agit du texte sur Oswald Spengler et Arnold Toynbee, écrit pour un public de philosophes, ce qui avait son importance :

"Que l'Histoire soit encore, dans l'assemblée des sciences humaines, une Cendrillon assise au bas bout de la table - nous savons bien pourquoi. Et nous savons aussi qu'elle participe à cette crise générale et profonde des idées et des conceptions scientifiques qu'a provoquée une poussée soudaine de certaines sciences : la physique en particulier, ébranlant des notions qui [...] semblaient acquises et sur lesquelles l'Humanité se reposait en toute quiétude. Nous savons qu'en fonction de telles transformations, et parce que la Science est une et toutes les sciences solidaires - nous savons que nos idées, fondées sur une philosophie scientifique périmée doivent être révisées [...] et nos méthodes en fonction de nos idées ${ }^{27}$."

Dans les années 1930, Bloch et Febvre établissaient leur méthode et menaient leur combat. Ce texte est donc une pièce rare, parce qu'il nous révèle combien le complexe continuait à agir à l'égard de la constitution épistémologiquement autonome de l'histoire. Celle-ci était encore une Cendrillon : tous les efforts de Berr depuis le début du siècle et le travail même des Annales depuis 1929 étaient restés isolés et n'avaient pas réussi à donner un statut solide de science à notre pauvre discipline, si maltraitée par l'assemblée des sciences humaines, alors que sur le plan des stratégies institutionnelles les choses ne se passaient pas trop mal, vu que Febvre avait été nommé au Collège de France en 1932, qu'il dirigeait l'Encyclopédie française, et que Bloch était nommé à la Sorbonne en 1936. Et l'histoire ne recevait pas encore l'imprimatur de «science» de la part des autres sciences, et notamment de la part des philosophes!

Mais le texte nous révèle aussi un autre aspect de la persistance du complexe d'infériorité : alors que la physique pouvait provoquer la crise, l'histoire en était incapable; elle devait plutôt «suivre» la réforme de ses sœurs plus importantes. Cela valait même si le complexe était parfaitement contrebalancé par la très louable conscience qu'avait Febvre de devoir réviser les idées en dépassant la «philosophie scientifique périmée » sur laquelle elles étaient fondées - tout en gardant l'optimisme très berrien sur la "Science une», dont on ne connaît pourtant

27. L. FeBVRe, op. cit. supra n. 14, p. 143. 
ni le statut ni le caractère, mais dont la validité était confortée par l'autorité toute philosophique de Rey.

Cette image de la science a donc empêché de reconnaître le fait apparemment très simple que l'histoire, telle qu'elle était pratiquée et théorisée par Bloch et Febvre, avait acquis un statut épistémologique (donc scientifique) très fort... mais que ce statut épistémologique était tellement nouveau et original par rapport à celui des sciences traditionnelles, et même des autres sciences humaines, qu'ils ne l'ont jamais considéré comme tel. Je me réfère à quelque chose de très connu, à savoir le caractère novateur du travail de Bloch et de Febvre à propos duquel on a fait couler beaucoup d'encre. En feuilletant leurs écrits publiés à partir des années 1930, l'ambiguïté par rapport à l'image traditionnelle de la science est de plus en plus diluée, alors même que persiste, toujours plus importante, la revendication méthodologique de leur nouvelle façon de travailler.

Les textes, si on les interprète de ce point de vue, sont clairs et on peut aisément $\mathrm{y}$ déceler une prise de position épistémologique très nette, qui aurait pu permettre aux historiens, en absence de complexes, de se présenter aux épistémologues et aux autres sciences sociales avec presque le même poids que des physiciens qui avaient réalisé une révolution théorique (thermodynamique, relativité, mécanique quantique). On ne doit pas oublier que, dans les années 1930, les rapports entre épistémologie et histoire des sciences étaient en train de changer profondément et allaient avoir des répercussions importantes dans la philosophie des sciences. Les idées de Bachelard, par exemple, auraient pu montrer à nos historiens que ce qu'ils étaient en train de faire était déjà une constitution épistémologique disciplinaire qui n'était certainement pas inférieure à celle des autres, et qu'elle pouvait même ouvrir des possibilités nouvelles pour les autres sciences.

Ainsi Bloch ouvrait l'histoire à l'étude des différences dans son essai sur l'histoire comparée qui, avec celui de Henri Pirenne de 1923, constitue un des points de repère les plus importants pour une nouvelle épistémologie de l'histoire ${ }^{28}$ : le classement et l'analyse, procédés caractéristiques d'un travail scientifique, ne devaient plus avoir comme but prioritaire l'identification et le rassemblement par ressemblance. La méthode comparative « justement conçue $[\ldots]$ porte au contraire un intérêt particulièrement vif à la perception des différences ». Bloch pousse en réalité son discours jusqu'à énoncer un principe qui était tout à fait épistémologique :

«Déterminer, non seulement en gros, que deux objets ne sont pas pareils, mais encore - besogne infiniment plus difficile, mais aussi beaucoup plus intéres-

28. Bloch s'en rendait parfaitement compte, et c'est pour cela qu'il choisit de donner à cet essai la première place dans son livre projeté en 1932, Historiens à l'atelier. 
sante - par quels caractères précis ils se distinguent, supposé évidemment, comme première démarche, qu' on les contemple tour à tour ${ }^{29}$. "

Seule la comparaison, disait Bloch, montre qu'il y a problème, et le «problème » était une autre des notions clés de la nouvelle démarche de l'histoire. C'est que la méthode comparative nous permettait de comprendre le sens de la pratique historienne, c'est-à-dire en quoi l'histoire était « une science des différences ». Cela voulait dire aussi que la réalité n’est pas simple, ni celle qu'on considère synchroniquement par la physique, par exemple, ni celle qu'on considère dans l'écoulement du temps. La conception de la réalité, réalité toujours humaine, même lorsqu'on parle de chimie ou de physique, puisque c'est l'homme qui la considère en ces termes, se faisait sous le signe de la complexité, non de la simplicité - et c'est sous le même signe de la complexité que Bachelard élaborait son épistémologie de la connaissance approchée et du nouvel esprit scientifique.

Différencier c'était rendre la complexité du réel. Et Febvre semblait enchaîner, du côté d'une épistémologie de la connaissance : "Pour l'historien [...] comprendre ce n'est pas clarifier, simplifier, réduire à un schéma logique parfaitement net [...] Comprendre, c'est compliquer. C'est enrichir en profondeur. C'est élargir de proche en proche. C'est mêler à la vie ${ }^{30}$. »

Et Bloch poursuivait encore : «L'homme, n'en doutons pas, change aussi, dans sa mentalité et, peut-être, jusque dans les profondeurs de son être physique ${ }^{31}$. »Cela n'empêche évidemment pas de chercher aussi les caractères invariants des phénomènes, ceux qui nous permettent d'établir des lois valables. Mais ils sont intégrés dans une vision plus articulée des choses et du temps. L'histoire joue à ce propos un rôle épistémologique qui devient de plus en plus important et présent dans la conscience de Bloch.

« Le cas des faits historiques - écrivait-il encore - ne diffère après tout que par le degré de celui que nous offrent tous les autres phénomènes de la nature. Jamais un phénomène ne se reproduit exactement pareil à lui-même. Ce qui, par contre, demeure semblable, ce sont certains facteurs dont la combinaison varie plus ou moins. Faut-il le répéter? faire varier ces facteurs de façon à en apprécier les effets, voilà en quoi précisément consiste une expérience [...] L'expérience naturelle qu'est l'expérience historique se trouve dans la même situation que les autres ${ }^{32}$. »

29. M. BLoch, « Pour une histoire comparée des sociétés européennes », art. cit. supra n. 20 , p. 27.

30. L. Febvre, op. cit. supra n. 14, p. 76.

31. M. BLoch, "Que demander à l'histoire?", 1937, repr. in Mélanges, op. cit. supra n. 20, p. 7. G. Bachelard s'était posé la même question et l'avait résolue : il avait acquis la conviction que « la science contemporaine crée une nouvelle nature, dans l'homme et hors de l'homme », voir G. BACHELARD, «Le nouvel esprit scientifique », Encyclopédie française, Paris, 1957, t. XIX, p. 19-14.15.

32. M. Bloch, art. cit. supra n. 31 , p. 10 (c'est moi qui souligne). 
Derrière ces mots de 1937 , on pourrait reconnaître encore tout un échafaudage épistémologique qu'il serait facile de démonter : mais il y a aussi un pas en avant décisif par rapport aux écrits de Febvre cités plus haut, car l'histoire était comparée cette fois aux autres sciences sur un même niveau épistémologique. Son statut scientifique, sa dignité de science, était donc en place - et nous savons que c'était aussi du fait de l'état de crise qui frappait le statut épistémologique des sciences naturelles - et ce depuis quelques années déjà.

La leçon inaugurale que Febvre tint en 1933 au Collège de France en était elle aussi un exemple éminent, de même que l'activité des Annales. Febvre se rendait parfaitement compte que leur « discours » était bouleversant pour les « traditionalistes », mais il était sûr qu'il n'en était pas ainsi pour les scientifiques. Il l'écrivait explicitement dans une lettre à Bloch le 26 décembre $1933^{33}$ : « [...] n'ignorons pas que ce que nous disons là heurte, choque, fait cabrer. Mais qui ? Pas les scientifiques qui trouvent que c'est monnaie courante. Or les scientifiques sont à gagner. »

Si nous regardons de près, le fait même que les scientifiques étaient censés être gagnés à la cause des Annales est par soi très significatif, et cela pas seulement du point de vue des stratégies et des alliances. Ils étaient à gagner parce que, du fait de la crise, ils pouvaient bien comprendre les propos de ceux qui prônaient une nouvelle attitude de la recherche historique - mais aussi de la recherche en général, ce "général », comme la "Science une », revient plusieurs fois sous la plume de Febvre. Les scientifiques pouvaient en outre saisir l'enjeu et le contenu épistémologique et pratique du combat de Febvre et de Bloch : pour eux, pas de problèmes, par exemple, pour concevoir les faits comme constructions intellectuelles, en reconnaissant le rôle essentiel qu'y jouait l'interprétation. Les deux directeurs des Annales insistaient beaucoup sur ce dernier point, car s'il y avait une discipline où cela était vrai, c'était l'histoire.

De là suivait naturellement l'histoire conçue sous le signe du problème, car interpréter n'était qu'un aspect du travail. L'autre aspect, le problème, était très complexe et ne pouvait pas être réduit simplement à la notion d'hypothèse. Là encore, la référence à Bachelard est nécessaire, puisque Le Nouvel Esprit scientifique représentait l'effort fait par l'épistémologue pour dépasser par une nouvelle problématique la place un peu restreinte que l'on accordait à l'hypothèse :

«Le doute universel (cartésien) pulvérisait irrémédiablement le donné en un amas de faits hétéroclites. Il ne correspond à aucune instance réelle de la

33. M. Bloch, L. FebVRe, Correspondance, op. cit. supra n. 19, p. 463. 
recherche scientifique. La recherche scientifique réclame, au lieu de la parade du doute universel, la constitution d'une problématique ${ }^{34}$. »

Tout le sens de la science était là. Et aussi de l'histoire, car Febvre ajoute : «C'est que, poser un problème, c'est précisément le commencement et la fin de toute histoire. Pas de problèmes, pas d'histoire ${ }^{35}$. »

L'épistémologie bachelardienne faisait donc le jeu de la « nouvelle histoire ", et inversement, puisque Febvre, en épistémologue, continuait ainsi :

«Or, rappelez-vous : si je n'ai point parlé de “ science ” de l'histoire, j’ai parlé "d'étude scientifiquement conduite". Ces deux mots n'étaient point là pour faire riche. "Scientifiquement conduite", la formule implique deux opérations, celles-là mêmes qui se trouvent à la base de tout travail scientifique moderne : poser des problèmes et formuler des hypothèses. »

Pour Febvre aussi, donc, le problème ne s'identifiait pas à l'hypothèse, ce qui du point de vue épistémologique n'est pas sans conséquences.

Il serait évidemment absurde d'exiger de Febvre ou de Bloch une thématisation épistémologique des termes utilisés, même si Bloch l'a ébauchée dans son Apologie pour l'histoire. Ni l'un ni l'autre n'étaient ni ne voulaient être des philosophes des sciences... mais ils faisaient usage de ces termes et de ces « outils » conceptuels, et cet usage était sans doute intéressant et créatif d'un point de vue philosophique ${ }^{36}$.

Ce tri des implications épistémologiques dans les écrits de Febvre et de Bloch pourrait être poursuivi longuement. D'autres notions y jouaient, en effet, des rôles très importants : celle de « subjectivité ", par exemple, ou d' « humanité », de « critique collective », d' « inquiétude de la recherche », de « possibilité », etc., pour en citer quelques-unes. Il n'en reste pas moins que le modèle épistémologique dominant auquel nos historiens se référaient n'était pas radicalement mis en question. Tout en utilisant dans leurs pratiques des procédés, des méthodes, des concepts et des idées véritablement novateurs, ils ne s'ouvraient pas à l'écoute d'épistémologues comme Bachelard et Koyré. Ainsi, ils ne se rendaient pas compte de la portée «philosophique » de leur travail et des conséquences qu'ils auraient pu provoquer dans l'assemblée des sciences humaines et naturelles. D'autre part, comme nous l'avons signalé, les épistémologues et les philosophes

34. G. Bachelard, Le Rationalisme appliqué, 1949, Paris, Presses universitaires de France, 1961, p. 51, et ID., Le Nouvel Esprit scientifique, Paris, Presses universitaires de France, 1934.

35. L. Febvre, op. cit. supra n. 14, p. 22.

36. Pour ce que j'en sais, seul G. Massicotte a essayé de trier les implications épistémologiques des cuvres de L. Febvre, in Guy MassicotTE, L'Histoire-problème, Saint-HyacinteParis, Edisem, 1981. 
étaient trop pris par leurs problèmes généraux pour s'apercevoir des potentialités qu'il y avait dans cette nouvelle façon de pratiquer et de penser l'histoire, et les historiens des sciences, qui auraient pu servir de trait d'union, étaient eux aussi trop enfermés dans la spécificité de leur objet disciplinaire.

En réalité, cette étrange rencontre n'a pas été manquée. Sur des plans et dans des temps différents, les idées ont circulé et les concepts ont été repris par l'une ou l'autre discipline. C'est pourquoi j'ai choisi de parler d'un « étrange théâtre ».

Un texte de Bloch semble remplir la fonction de conclusion, car il rassemble, en quelque sorte, tous les éléments présentés jusqu'ici. Selon ce texte, l'histoire était en fait dotée depuis longtemps d'un statut scientifique (épistémologique) autonome et originel, qui n'aurait pas dû la faire sentir « inférieure » aux autres sciences; mais, en même temps, il recèle encore, dans les métaphores employées, l'écho du complexe dont il a été question plus haut. Ces phrases, dans leur optimisme, font certes état de la crise du savoir de l'époque, mais elles y réagissent de façon constructive, ouvrant à nouveau la voie à une épistémologie capable de ne pas se limiter simplement à l'histoire, chose qui reste encore à faire :

« Nous sommes donc, désormais, beaucoup mieux préparés à admettre que, pour ne pas s'avérer capables de démonstrations euclidiennes ou d'immuables lois de répétition, une connaissance puisse, néanmoins, prétendre au nom de scientifique. Nous acceptons beaucoup plus aisément de faire de la certitude et de l'universalisme une question de degré. Nous ne nous sentons plus l'obligation de chercher à imposer à tous les objets du savoir un modèle intellectuel uniforme, emprunté aux sciences de la nature physique; puisque là même, ce gabarit a cessé de s'appliquer tout entier. Nous ne savons pas encore très bien ce que seront un jour les sciences de l'homme. Nous savons que pour être tout en continuant, cela va de soi, d'obéir aux règles fondamentales de la raison - elles n'auront pas besoin de renoncer à leur originalité, ni d'en avoir honte ${ }^{37}$.

Enrico Castelli Gattinara

(mars 1996).

37. M. BLoch, Apologie pour l'histoire, Paris, Armand Colin, 1993, p. 77-78. 